\title{
Dynamics of the electrically induced insulator-to-metal transition in rare-earth nickelates
}

\author{
Javier del Valle $\odot,{ }^{1, *}$ Rodolfo Rocco $\odot,{ }^{2}$ Claribel Domínguez, ${ }^{1}$ Jennifer Fowlie $\odot,{ }^{1}$ Stefano Gariglio, ${ }^{1}$ \\ Marcelo J. Rozenberg, ${ }^{2}$ and Jean-Marc Triscone ${ }^{1}$ \\ ${ }^{1}$ Department of Quantum Matter Physics, University of Geneva, 24 Quai Ernest-Ansermet, 1211 Geneva, Switzerland \\ ${ }^{2}$ Laboratoire de Physique des Solides, UMR8502 CNRS-Université Paris-Sud, Université Paris-Saclay, 91405 Orsay Cedex, France
}

(Received 9 July 2021; revised 4 October 2021; accepted 4 October 2021; published 22 October 2021)

\begin{abstract}
Rare-earth nickelates feature an insulator-to-metal transition (IMT) that can be electrically triggered. We study the dynamics of this electrically induced transition by comparing the time-dependent transport properties of two distinct members of the $R e \mathrm{NiO}_{3}$ family: $\mathrm{NdNiO}_{3}$ and $\mathrm{SmNiO}_{3}$. We report stark differences in the nucleation and growth of the metallic phase, which evolve more rapidly for $\mathrm{NdNiO}_{3}$. With the aid of simulations, we identify the amplitude of the resistivity change across the IMT as the key parameter controlling the switching speed. Our results are in accordance with recent experiments in the $\mathrm{VO}_{x}$ family, contributing to a unified vision of the field-induced IMT dynamics across different families of correlated oxides.
\end{abstract}

DOI: 10.1103/PhysRevB.104.165141

\section{INTRODUCTION}

Certain materials, such as $\mathrm{VO}_{2}, \mathrm{~V}_{2} \mathrm{O}_{3}, \mathrm{NbO}_{2}, \mathrm{NiS}_{2-x} \mathrm{Se}_{x}$, or $\mathrm{GaTa}_{4} \mathrm{Se}_{8}$ [1-8], feature insulator-to-metal transitions (IMTs) that can be electrically triggered using a simple twoelectrode configuration. Upon application of a high enough voltage, they undergo a transition from insulator to metal, returning to the insulating phase when the voltage is removed. This yields a volatile resistive switching that has recently attracted a lot of attention both for its fundamental interest and for its potential application in emerging technologies such as neuromorphic computing or optoelectronics [9-19]. While the underlying physics of this transition has been intensely researched over the last decade, key aspects of it remain poorly understood. One of them is the transition dynamics, i.e., how do metallic domains nucleate and grow when a voltage is applied, and what are the parameters that govern their evolution? A recent work has provided a glimpse into this process by capturing the voltage-triggered IMT both with space and time resolution [20]. It shows that switching is initiated by the nucleation of small metallic hotspots that focus current, starting a runaway effect that leads to the percolation of a filament connecting the electrodes [7,21-23]. The resistance change across the IMT is suggested as the most important parameter controlling the metallic phase growth rate.

The study, however, was limited to $\mathrm{VO}_{2}, \mathrm{~V}_{2} \mathrm{O}_{3}$, and $\mathrm{V}_{3} \mathrm{O}_{5}$, which are Mott insulators with strong $d$-d correlations [24]. It is unclear whether those results can be extended to other systems featuring an IMT of different origin. One such system

\footnotetext{
*Corresponding author: javier.delvalle@unige.ch
}

Published by the American Physical Society under the terms of the Creative Commons Attribution 4.0 International license. Further distribution of this work must maintain attribution to the author(s) and the published article's title, journal citation, and DOI. is the rare-earth nickelates $\left(R e \mathrm{NiO}_{3}\right.$ where $R e$ can be most of the lanthanide rare earth elements) [25-27]. In contrast with the vanadates, $R e \mathrm{NiO}_{3}$ are charge-transfer oxides with strong hybridization between the $\mathrm{Ni} d$ and $\mathrm{O} p$ orbitals [28,29], resulting in more itinerant valence electrons and lower onsite Coulomb repulsion $[30,31]$. The transition into the insulating state is accompanied by a bond disproportionation of the $\mathrm{NiO}_{6}$ octahedra [32,33]. Electron-lattice coupling seems to play a fundamental role [34,35], and some works have interpreted this IMT as a magnetically assisted Peierls instability [36].

Despite the wide attention the $R e \mathrm{NiO}_{3}$ IMT has received in recent years, the electrically triggered IMT has barely been explored, with only two works that we are aware of [37,38]. In this paper, we study the switching dynamics of $\mathrm{NdNiO}_{3}$ and $\mathrm{SmNiO}_{3}$ by analyzing their time-dependent transport properties. We find that, in $\mathrm{NdNiO}_{3}$, metallization nucleates and grows at a much faster rate and has a stronger voltage dependence than in $\mathrm{SmNiO}_{3}$. With the help of resistor network simulations, we interpret these results considering only Joule heating and the resistivity ratio between the insulating and metallic states: a higher ratio focuses current into smaller regions and accelerates the metallization process. Our results resemble and support recent findings in the vanadate family [20] — despite the big differences in microscopic details between both oxide families - and contributes to a generalized view of the switching dynamics based on simple mesoscopic arguments.

\section{SAMPLES AND DIRECT CURRENT MEASUREMENTS}

$R e \mathrm{NiO}_{3}$ compounds do not have a cubic perovskite structure. Instead, $\mathrm{NiO}_{6}$ octahedra are slightly rotated with respect to their neighbors, resulting in $\mathrm{Ni}-\mathrm{O}-\mathrm{Ni}$ bond angles $<180^{\circ}$ [27]. This distortion is smaller and the bond angle greater (closer to $180^{\circ}$ ) the larger the rare earth radius. A smaller distortion increases orbital overlap and bandwidth, reducing the IMT temperature $[26,27,39]$. The transition temperature is 
lowest for $R e=\mathrm{Pr}$, gradually increasing for $R e=\mathrm{Nd}, \mathrm{Sm}$, $\mathrm{Eu}, \mathrm{Gd}$, Dy, Ho, etc. For $\mathrm{PrNiO}_{3}$ and $\mathrm{NdNiO}_{3}$, the IMT is sharp, first order [40-42], and concomitant with a magnetic transition from an antiferromagnetic insulator into a paramagnetic metal. For the other $\mathrm{ReNiO}_{3}$ compounds, the transition is smoother, resembling a second-order transition and with no magnetic ordering at the IMT (although antiferromagnetism appears at lower temperatures) [27]. In this paper, we investigated one oxide from each of the two types described above: $\mathrm{NdNiO}_{3}$ and $\mathrm{SmNiO}_{3}$. Both oxide films were grown on (001)-oriented $\mathrm{LaAlO}_{3}$ substrates using off-axis magnetron sputtering in an $\mathrm{Ar}: \mathrm{O}_{2}$ (3.5:1) mixture at a pressure of 180 mTorr. The substrate temperature was $460^{\circ} \mathrm{C}$. The films were $\sim 30 \mathrm{~nm}$ and grown epitaxially, as can be seen, using x-ray diffraction (Fig. S1 in the Supplemental Material [43]; see also [44]). Figure 1(a) shows resistivity vs temperature for both films. $\mathrm{NdNiO}_{3}$ shows a sharp hysteretic transition at $\sim 100 \mathrm{~K}$, with a resistivity change of two orders of magnitude. $\mathrm{SmNiO}_{3}$ has a much smoother transition at $\sim 375 \mathrm{~K}$, with one order of magnitude resistivity change. The differences in IMT can be better appreciated in Fig. S2 in the Supplemental Material [43], where the $\mathrm{NdNiO}_{3}$ transition is shifted by $250 \mathrm{~K}$.

To electrically trigger the IMT, Pt electrodes were patterned on top of the nickelate films using a combination of e-beam and optical lithography. Electrode separation was $300-400 \mathrm{~nm}$, as can be seen in Fig. 1(b). Figures 1(c) and 1(e) show the direct current (dc) voltage vs current characteristics of $\mathrm{NdNiO}_{3}$ and $\mathrm{SmNiO}_{3}$ nanodevices for different temperatures. Volatile resistive switching, caused by a transition into the metallic state, is readily visible for all temperatures shown. For $\mathrm{SmNiO}_{3}$, temperatures $<200 \mathrm{~K}$ required voltages $\sim 10 \mathrm{~V}$ that produced nonvolatile resistive switching, likely due to ion migration, as previously reported [45-50]. We must underline that the focus of this paper is the volatile resistive switching caused by the IMT, not nonvolatile effects due to ion migration and other types of electroforming [16,45-50]. There are stark differences in the switching properties of both oxides. $\mathrm{NdNiO}_{3}$ features a sudden, discontinuous jump above a threshold voltage $\left(V_{\mathrm{Th}}\right)$, together with a marked hysteresis in the $V-I$ curves. In contrast, $\mathrm{SmNiO}_{3}$ displays a continuous evolution from a high to a low resistance state. Rather than a clear threshold switching, a negative differential resistance (NDR) can be observed when a voltage $V_{\mathrm{NDR}}$ is reached. In both cases, switching becomes sharper as the temperature is lowered, with $\mathrm{SmNiO}_{3}$ even showing small jumps and hysteretic behavior.

\section{TIME-DEPENDENT CHARACTERIZATION}

We characterized the time-dependent response of our devices to an applied voltage. We used a function generator to create a long square voltage pulse that was applied to one of the electrodes of the sample, while the other electrode was connected to a $50 \Omega$ impedance channel of a Tektronix MSO064 oscilloscope. Thus, the oscilloscope gave a direct readout of the current going through the device as a function of time. The top panel in Fig. 2(a) shows current vs time in a $\mathrm{NdNiO}_{3}$ nanodevice at $4.2 \mathrm{~K}$, when different voltages are applied at $t=0$. For low voltages, resistance remains high and current low, meaning the IMT is not triggered. However, the (a)

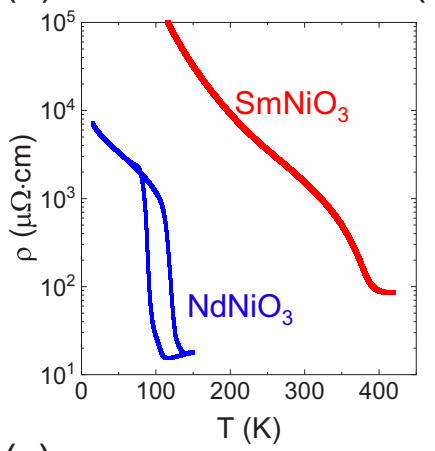

(c)

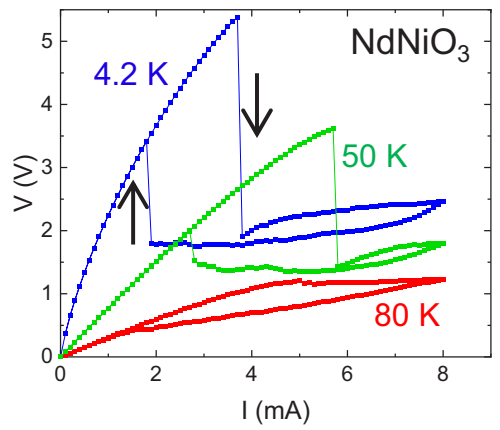

(e)

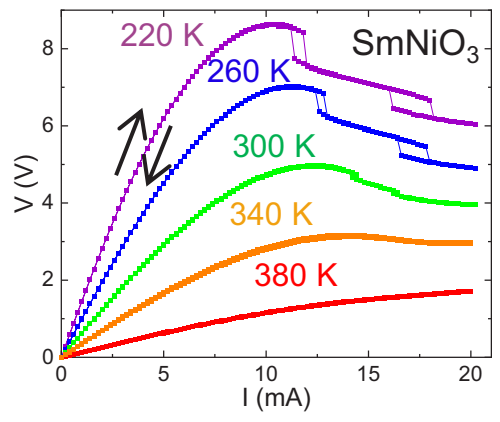

(b)

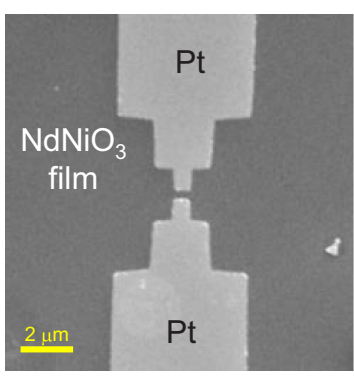

(d)

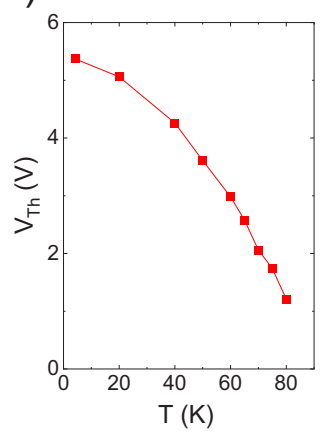

(f)

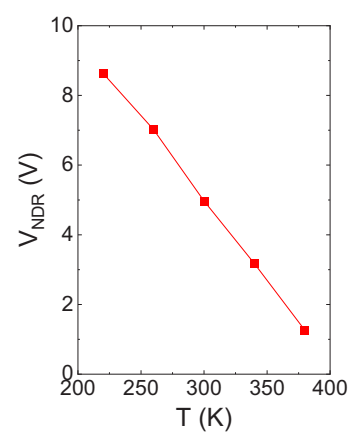

FIG. 1. (a) Resistivity vs temperature for $\mathrm{NdNiO}_{3}$ (blue) and $\mathrm{SmNiO}_{3}$ (red) measured in unpatterned thin films using the van der Pauw method. (b) Scanning electron microscopy image of one of the $\mathrm{NdNiO}_{3}$ nanodevices. (c) Direct current (dc) voltage vs current characteristics in $\mathrm{NdNiO}_{3}$. (d) Threshold voltage $\left(V_{\mathrm{Th}}\right)$ vs temperature for $\mathrm{NdNiO}_{3}$. (e) dc voltage vs current characteristics in $\mathrm{SmNiO}_{3}$. (f) Negative differential resistance voltage $\left(V_{\mathrm{NDR}}\right)$ for $\mathrm{SmNiO}_{3}$.

response of the sample drastically changes when a threshold voltage is crossed: while for $5.50 \mathrm{~V}$, the sample remains insulating for over $1 \mathrm{~ms}$, for $5.55 \mathrm{~V}$, a transition into the metallic state is triggered after just a few nanoseconds. The figure inset provides a closeup view of the first nanosecond after the voltage is applied, showing switching times as fast as $2-3 \mathrm{~ns}$, close to the experimental rise time of our setup.

The bottom panel of Fig. 2(a) corresponds to the same device but at a temperature of $60 \mathrm{~K}$, showing a rather different behavior. Instead of a sudden change, the response of the sample evolves more gradually as the amplitude of the applied voltage is increased. For low voltages, the IMT is not induced, but the threshold is less clear. For 3.7 and $3.8 \mathrm{~V}$, a transition into the metallic phase is triggered but only after a certain 
(a)
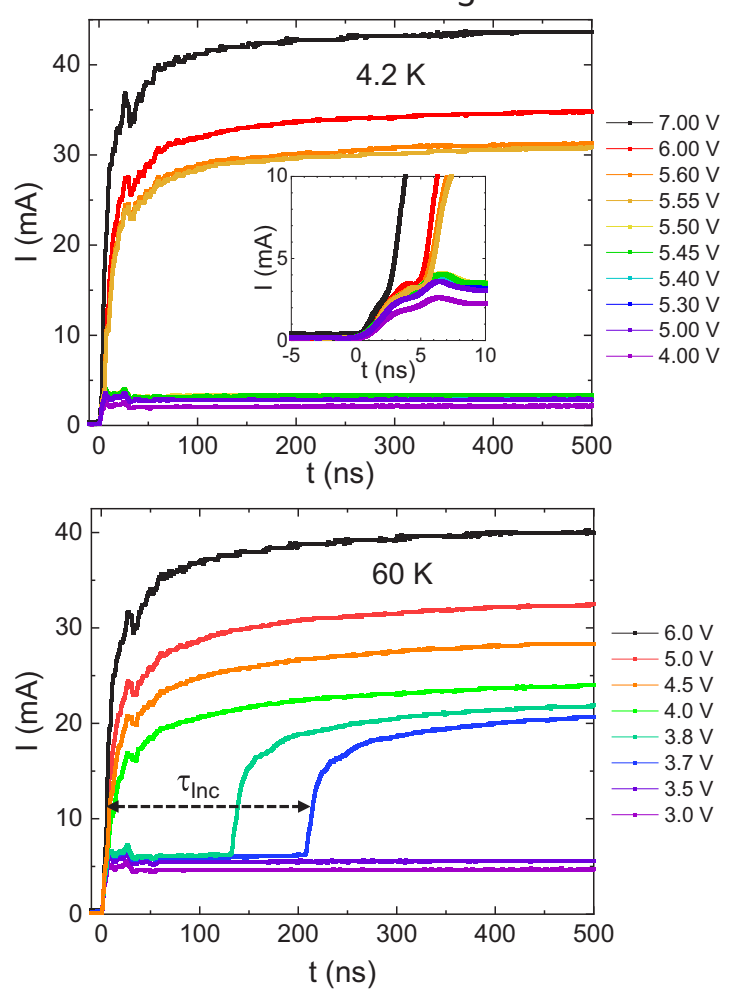

(b)

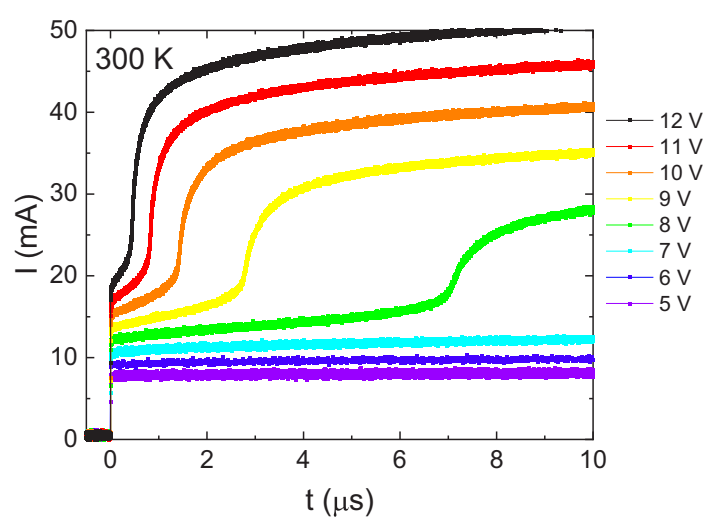

FIG. 2. Current vs time when voltage pulses of different amplitude are applied at $t=0$ for (a) $\mathrm{NdNiO}_{3}$ at $4.2 \mathrm{~K}$ (top panel) and $60 \mathrm{~K}$ (bottom panel), (b) $\mathrm{SmNiO}_{3}$ at $300 \mathrm{~K}$. Inset to (a): Magnified plot of the first nanoseconds after the voltage is applied in $\mathrm{NdNiO}_{3}$. $T=4.2 \mathrm{~K}$. At first glance, $\mathrm{NdNiO}_{3}$ at $60 \mathrm{~K}$ and $\mathrm{SmNiO}_{3}$ at $300 \mathrm{~K}$ might look very similar, but as the time axis shows, switching is $\sim 10$ times faster for $\mathrm{NdNiO}_{3}$.

incubation time $\tau_{\text {Inc }}$ has passed. This $\tau_{\text {Inc }}$ is shorter the higher the applied voltage. Voltage dependence is even smoother for $\mathrm{SmNiO}_{3}$ devices, as shown in Fig. 2(b). In this case, not only does $\tau_{\text {Inc }}$ evolve very gradually with voltage, but the transition from insulator to metal is itself less well defined. In the current vs time curves, a positive slope within the insulating state is visible, making the IMT more blurred. It is also important to note that switching dynamics are much slower for $\mathrm{SmNiO}_{3}$ than $\mathrm{NdNiO}_{3}$.

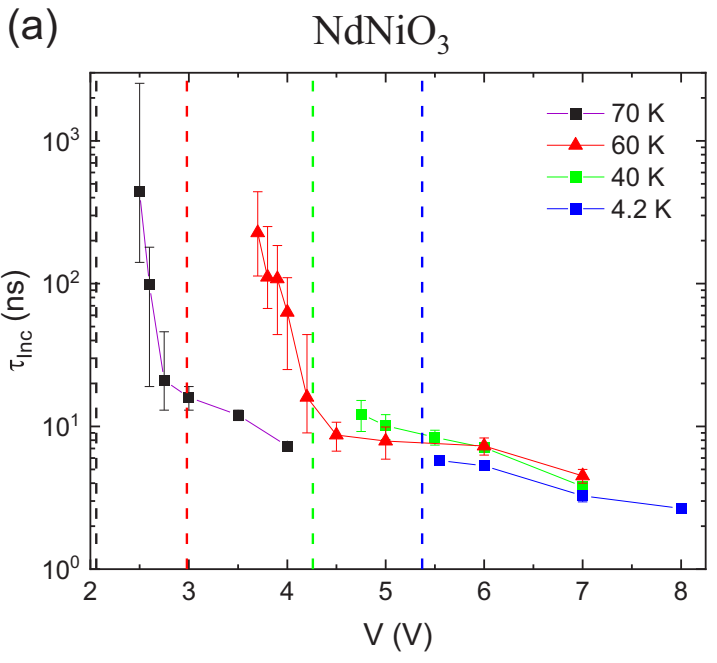

(b) $\quad \mathrm{SmNiO}_{3}$

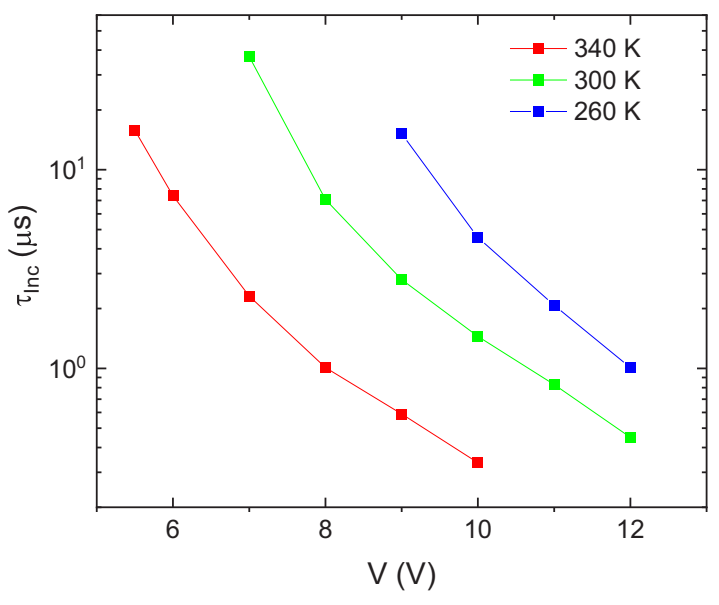

FIG. 3. Incubation time ( $\tau_{\text {Inc }}$ ) vs pulse voltage for (a) $\mathrm{NdNiO}_{3}$ and (b) $\mathrm{SmNiO}_{3}$. Several temperatures are shown in each case. The vertical dashed lines in panel (a) correspond to the $V_{\text {Th }}$ plotted in Fig. 1(d).

Figure 3 summarizes the above observations by showing $\tau_{\text {Inc }}$ vs voltage for several temperatures for both samples. The $\tau_{\text {Inc }}$ axis is in logarithmic scale. The plot shows that switching dynamics depend drastically on the applied voltage, i.e., small voltage changes lead to large variations in the sample response. From Fig. 3, several important points can be underlined: (i) switching is much faster for $\mathrm{NdNiO}_{3}$, (ii) voltage dependence is stronger for $\mathrm{NdNiO}_{3}$, and (iii) voltage dependence is stronger at lower temperatures. The latter can be better appreciated for $\mathrm{SmNiO}_{3}$ in Fig. S3 in the Supplemental Material [43], where current vs time curves at 340 and $260 \mathrm{~K}$ are compared. The contrast in dynamic response resembles the differences in the dc $V-I$ characteristics shown in Fig. 1: $\mathrm{NdNiO}_{3}$ has a sharp resistance drop above a well-defined $V_{\mathrm{Th}}$, while $\mathrm{SmNiO}_{3}$ does not show a clear threshold but a rather continuous change. For both samples, voltage-current curves become more abrupt as the temperature is lowered.

We also studied the volatile character of this resistive switching. We did so by measuring how fast the insulating state recovers after the applied voltage is removed (Fig. S4 
in the Supplemental Material [43]). We find that most of the sample relaxes to the insulating state within a few microseconds. This relaxation is faster the lower the temperature, the opposite of what would be expected if oxygen vacancies played a major role in the switching process [51]. This means that the recovery time is likely set by the cool down and the phase relaxation dynamics (further discussion in the Supplemental Material [43]). The effect of electrode geometry was also explored, finding that larger separations lead to higher switching voltages and slower dynamics (see discussion and Fig. S5 in the Supplemental Material [43]).

\section{DISCUSSION AND RESISTOR NETWORK SIMULATIONS}

These results can be understood considering the nucleation and growth dynamics of the metallic phase. A recent work has provided an experimental account of this process using a combination of transport and reflectivity measurements [20]. It was shown that, starting from a homogeneously insulating system at $t=0$, inhomogeneities appear as soon as the voltage is applied. These inhomogeneities are hotspots with lower resistivity that concentrate current, locally increasing Joule heating. This lowers resistivity even more, creating a positive feedback loop that eventually leads to the formation of a metallic filament. This final filament configuration had been previously revealed in steady-state experiments [7,2123]. The temperature resistivity change across the IMT is expected to be a crucial parameter controlling the feedback loop and, hence, the switching speed. This is depicted in Fig. 4(a): a larger $\rho_{\text {Ins }} / \rho_{\text {Met }}$ will concentrate more current into any hypothetical hotspot, increasing local heating and consequently accelerating the transition from insulator to metal.

To better visualize this, we performed numerical simulations, modeling our devices as a two-dimensional network of resistors in thermal contact with a substrate at temperature $T[3,6,20,52,53]$. Each node in the network can be either metallic or insulating (Fig. S6 in the Supplemental Material [43]). The metallic resistivity is assumed constant $\left(\rho_{\mathrm{Met}}\right)$, while the insulating resistivity has an Arrhenius temperature dependence (with $\rho=\rho_{\text {Ins }}$ at $T=T_{\mathrm{IMT}}$ ). The state of each cell is governed by a Landau type free energy functional that mimics a first-order transition. At $t=0$, a voltage is applied. For each simulation step, the current and temperature distributions are calculated, and the state of each cell is updated with a probability computed from the reevaluated energy barrier, allowing us to explore the switching dynamics. More details on the simulations can be found in the Supplemental Material [43]. Figure 4(b) shows the two-dimensional temperature and resistivity maps at the precise moment when a metallic filament percolates between both electrodes, which can be identified as a sudden and sharp drop in resistance. Three different temperatures are shown: $0.18,0.35$, and $0.88 T_{\mathrm{IMT}}$. Note that filaments form in the electrode corners since we do not include intrinsic defects, and those are the points of maximum electric field. A clear trend is visible: as the temperature is lowered, the resistivity of the insulating state becomes higher, confining nucleation of metallic domains to smaller areas and markedly increasing Joule heating. A higher local temperature would translate into faster metallization and switching times. (a)

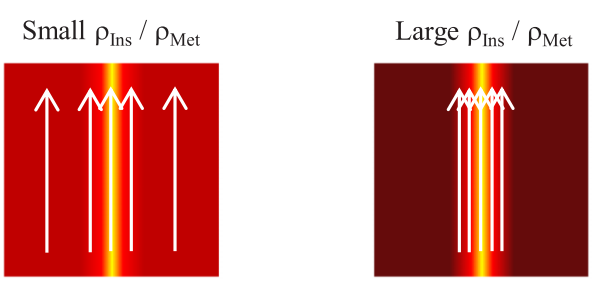

(b)
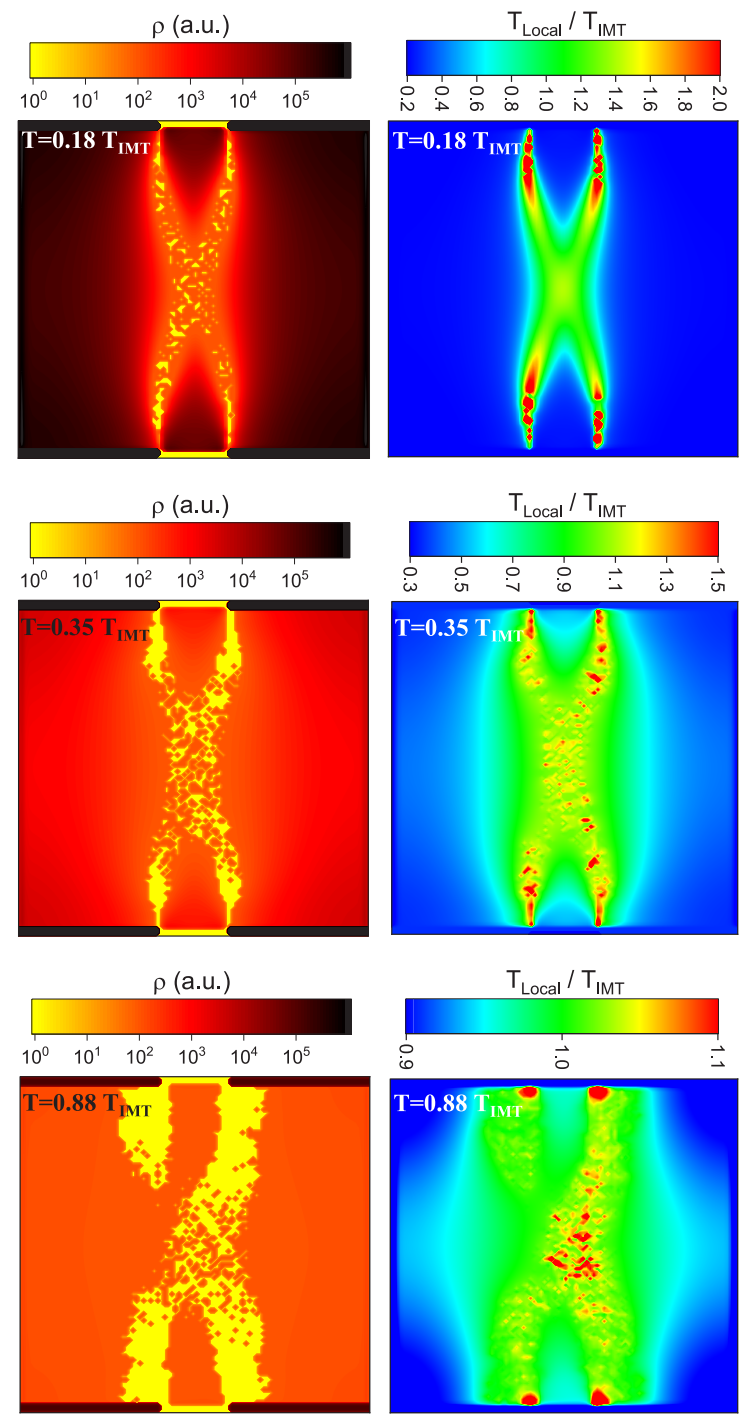

FIG. 4. (a) Schematic representation of current focusing in an inhomogeneous system with an insulator-to-metal transition (IMT) of magnitude $\rho_{\mathrm{Ins}} / \rho_{\mathrm{Met}}$. Current is focused into smaller areas when the $\rho_{\text {Ins }} / \rho_{\text {Met }}$ ratio is large. (b) Two-dimensional (2D) plots of the resistivity (left columns) and temperature (right columns) distributions obtained with resistor network simulations at the moment in which percolation takes place. Resistivity is color-coded in a logarithmic scale, while temperature is color-coded in linear scale. Note that the resistivity scale is the same for all plots, but the temperature is different for each panel to better appreciate the individual details in each case. Three different base temperatures are shown: $0.18 T_{\mathrm{IMT}}$ (top row), $0.35 T_{\mathrm{IMT}}$ (middle row), and $0.88 T_{\mathrm{IMT}}$ (bottom row). $T_{\mathrm{IMT}}$ is the IMT transition temperature. The $\rho_{\text {Ins }} / \rho_{\text {Met }}$ ratio is $10^{2}$ (larger and smaller ratios can be seen in the Supplemental Material [43]). 
This trend is not only visible as the temperature is lowered but also as $\rho_{\text {Ins }} / \rho_{\text {Met }}$, the resistivity jump across the IMT, is increased (Fig. S7 in the Supplemental Material [43]).

The simulation results explain the data in Fig. 3. For high $\rho_{\text {Ins }} / \rho_{\text {Met }}$, either the voltage is not high enough to induce any metallization or, if it does, complete switching into the metallic state is very fast. This all-or-nothing behavior is very clear for $\mathrm{NdNiO}_{3}$ at $4.2 \mathrm{~K}$, becoming more gradual as the temperature is increased. $\mathrm{SmNiO}_{3}$ features a much smoother IMT with lower $\rho_{\text {Ins }} / \rho_{\text {Met }}$. As a result, current confinement and switching speed are greatly reduced. Interestingly, a similar trend is observed in the vanadates, where $\mathrm{V}_{2} \mathrm{O}_{3}$ has larger resistivity change across the IMT than $\mathrm{VO}_{2}$ and much larger than $\mathrm{V}_{3} \mathrm{O}_{5}$. As a result, $\mathrm{V}_{2} \mathrm{O}_{3}$ switches electrically faster than $\mathrm{VO}_{2}$ and much faster than $\mathrm{V}_{3} \mathrm{O}_{5}[20]$.

A similar $\rho_{\text {Ins }} / \rho_{\text {Met }}$ trend in two very different oxide families (vanadates and nickelates) suggests that the mechanism proposed here could apply to all systems featuring IMTs. Our model is based on very simple and general assumptions, applicable to any material. Considering this, $\mathrm{ReNiO}_{3}$ with smooth, second-order transitions, such as $\mathrm{EuNiO}_{3}, \mathrm{GdNiO}_{3}$, or $\mathrm{DyNiO}_{3}$ should display slower switching and smoother $I-V$ properties than $\mathrm{PrNiO}_{3}$ or $\mathrm{NdNiO}_{3}$, which have sharp, firstorder IMTs. Similarly, switching dynamics should depend strongly on any external parameter that influences the $R$ vs $T$ of the material. In the $R e \mathrm{NiO}_{3}$ case, strain is known to play a major role $[39,54,55]$. For instance, compressive strain has been shown to lower the transition temperature and increase $\rho_{\text {Ins }} / \rho_{\text {Met }}$ across the IMT in $\mathrm{SmNiO}_{3}$ [39]. This would lead to faster switching dynamics and sharper $I-V$, resembling the $\mathrm{NdNiO}_{3}$ results shown here. Conversely, our $\mathrm{NdNiO}_{3}$ films are under a small compressive strain $(-0.5 \%)$ imposed by the $\mathrm{LaAlO}_{3}$ substrate, which lowers the IMT temperature and increases $\rho_{\text {Ins }} / \rho_{\text {Met }}$ [54]. Based on our results, we expect unstrained $\mathrm{NdNiO}_{3}$ to show slower switching dynamics, more like the $\mathrm{SmNiO}_{3}$ behavior shown here.

This mechanism also explains the presence of small jumps and hysteresis in the dc $V-I$ properties of $\mathrm{SmNiO}_{3}$ at low temperatures [Fig. 1(e)]: a larger $\rho_{\text {Ins }} / \rho_{\text {Met }}$ ratio favors runaway effects, creating current/voltage discontinuities when performing dc measurements. The observation of a hysteresis does not necessarily imply a first-order character in the $\mathrm{SmNiO}_{3} \mathrm{IMT}$. A hysteresis may arise purely from an asymmetry in the distribution of metallic/insulating domains when the current is ramped up and down. The system is homogeneous when the current is ramped up, but it has a metallic filament that concentrates Joule heating when it is ramped down. This makes it harder for insulating domains to nucleate, yielding a hysteresis that becomes more apparent as temperature is decreased. A detailed description of the dynamics that give rise to voltage discontinuities and hysteresis during the electrical triggering of a second-order IMT can be found in the work of Kumar et al. [10].

We must note that we have considered Joule heating as the only underlying mechanism that induces the electrically triggered IMT in our $R e \mathrm{NiO}_{3}$ devices. It is the most straightforward explanation and has already been shown to induce the IMT in systems such as $\mathrm{NbO}_{2}$ or $\mathrm{VO}_{2}[5,56]$. The insulating state resistivity of $\mathrm{ReNiO}_{3}$ is three to four orders of magnitude lower than that of $\mathrm{VO}_{2}$ [57], and the current density $(J)$ just before our devices switch is in the order of $10^{10}$ to $10^{11} \mathrm{~A} / \mathrm{cm}^{2}$, two orders of magnitude larger than what has been reported for $\mathrm{VO}_{2}$ [3,58]. Considering that, in a homogeneous system-before filament formation-dissipation is proportional to $\rho_{\text {Ins }} \cdot J^{2}$, it is very likely that Joule heating is indeed inducing the IMT in $\mathrm{NdNiO}_{3}$ and $\mathrm{SmNiO}_{3}$, as was suggested before [37]. Another possible mechanism would be a direct field effect triggering of the IMT due to carrier injection and destabilization of the insulating state $[59,60]$, as has been observed in $\mathrm{V}_{2} \mathrm{O}_{3}$ [61,62]. However, $\mathrm{V}_{2} \mathrm{O}_{3}$ has an insulating state resistivity five to seven orders of magnitude higher than that of $\mathrm{ReNiO}_{3}$ [57], which largely precludes any Joule heating effect. Given the comparatively low resistivities of $\mathrm{NdNiO}_{3}$ and $\mathrm{SmNiO}_{3}$, it is a rather unlikely scenario.

\section{CONCLUSIONS}

In conclusion, we have studied the dynamics of the electrically triggered IMT in $\mathrm{NdNiO}_{3}$ and $\mathrm{SmNiO}_{3}$. We did so by analyzing the time-dependent transport properties of our devices upon the application of a voltage pulse. We observed that switching times $\left(\tau_{\text {Inc }}\right)$ are much shorter for $\mathrm{NdNiO}_{3}$ than for $\mathrm{SmNiO}_{3}$. We found that $\tau_{\text {Inc }}$ depends strongly on the applied voltage, that dependence being more dramatic for $\mathrm{NdNiO}_{3}$, and for lower temperatures. Our results can be interpreted considering the resistivity ratios between the insulating and metallic states. We use resistor network simulations to show that a higher $\rho_{\text {Ins }} / \rho_{\text {Met }}$ ratio results in a more confined distribution of metallic domains during nucleation, increasing local Joule heating and accelerating the metallization process. We compare two very different and representative members of the rare-earth nickelates, offering a hint on what could be expected for the rest of the $R e \mathrm{NiO}_{3}$ compounds. Our work in this paper agrees very well with recent observations in the vanadate family [20], suggesting that the dynamics of the electrically triggered IMT can be explained using simple mesoscopic arguments, independent from microscopic details and applicable to very different systems that feature an IMT.

\section{ACKNOWLEDGMENTS}

The authors thank Marco Lopes, Ignacio Gutierrez Lezama, Alberto Morpurgo, Iaroslav Gaponenko, and Patrycja Paruch for their support during the fabrication and measurement of these samples. This paper was funded by the Swiss National Science Foundation through an Ambizione Fellowship (\#PZ00P2_185848). The oxide growth was supported by the European Research Council under the European Union's Seventh Framework Program (FP7/2007-2013)/ERC Grant Agreement 319286 Q-MAC and the Swiss National Science Foundation Project No. 200020-179155. MR and RR acknowledge support of the French ANR project "MoMA" ANR-19-CE30-0020. 
[1] G. Stefanovich, A. Pergament, and D. Stefanovich, Electrical switching and Mott transition in $\mathrm{VO}_{2}$, J. Phys. Condens. Matter. 12, 8837 (2000).

[2] H. T. Kim, B. G. Chae, D. H. Youn, S. L. Maeng, G. Kim, K. Y. Kang, and Y. S. Lim, Mechanism and observation of Mott transition in $\mathrm{VO}_{2}$-based two- and three-terminal devices, New J. Phys. 6, 52 (2004).

[3] J. del Valle, P. Salev, F. Tesler, N. M. Vargas, Y. Kalcheim, P. Wang, J. Trastoy, M. H. Lee, G. Kassabian, J. G. Ramírez, M. J. Rozenberg, and I. K. Schuller, Subthreshold firing in Mott nanodevices, Nature (London) 569, 388 (2019).

[4] J. S. Brockman, L. Gao, B. Hughes, C. T. Rettner, M. G. Samant, K. P. Roche, and S. S. P. Parkin, Subnanosecond incubation times for electric-field-induced metallization of a correlated electron oxide, Nat. Nanotechnol. 9, 453 (2014).

[5] Z. Wang, S. Kumar, Y. Nishi, and H. S. P. Wong, Transient dynamics of $\mathrm{NbO}_{x}$ threshold switches explained by Poole-Frenkel based thermal feedback mechanism, Appl. Phys. Lett. 112, 193503 (2018).

[6] P. Stoliar, L. Cario, E. Janod, B. Corraze, C. Guillot-Deudon, S. Salmon-Bourmand, V. Guiot, J. Tranchant, and M. Rozenberg, Universal electric-field-driven resistive transition in narrow-gap Mott insulators, Adv. Mater. 25, 3222 (2013).

[7] H. Madan, M. Jerry, A. Pogrebnyakov, T. Mayer, and S. Datta, Quantitative mapping of phase coexistence in mott-Peierls insulator during electronic and thermally driven phase transition, ACS Nano 9, 2009 (2015).

[8] V. Guiot, L. Cario, E. Janod, B. Corraze, V. Ta Phuoc, M. Rozenberg, P. Stoliar, T. Cren, and D. Roditchev, Avalanche breakdown in $\mathrm{GaTa}_{4} \mathrm{Se}_{8-x} \mathrm{Te}_{x}$ narrow-gap Mott insulators, Nat. Commun. 4, 1722 (2013).

[9] S. M. Bohaichuk, S. Kumar, G. Pitner, C. J. McClellan, J. Jeong, M. G. Samant, H. S. P. Wong, S. S. P. Parkin, R. S. Williams, and E. Pop, Fast spiking of a Mott $\mathrm{VO}_{2}$-carbon nanotube composite device, Nano Lett. 19, 6751 (2019).

[10] S. Kumar, J. P. Strachan, and R. S. Williams, Chaotic dynamics in nanoscale $\mathrm{NbO}_{2}$ Mott memristors for analogue computing, Nature (London) 548, 318 (2017).

[11] J. del Valle, P. Salev, Y. Kalcheim, and I. K. Schuller, A caloritronics-based Mott neuristor, Sci. Rep. 10, 4292 (2020).

[12] M. D. Pickett, G. Medeiros-Ribeiro, and R. S. Williams, A scalable neuristor built with Mott memristors, Nat. Mater. 12, 114 (2013).

[13] W. Yi, K. K. Tsang, S. K. Lam, X. Bai, J. A. Crowell, and E. A. Flores, Biological plausibility and stochasticity in scalable $\mathrm{VO}_{2}$ active memristor neurons, Nat. Commun. 9, 4661 (2018).

[14] N. A. Butakov, M. W. Knight, T. Lewi, P. P. Iyer, D. Higgs, H. T. Chorsi, J. Trastoy, J. Del Valle Granda, I. Valmianski, C. Urban, Y. Kalcheim, P. Y. Wang, P. W. C. Hon, I. K. Schuller, and J. A. Schuller, Broadband electrically tunable dielectric resonators using metal-insulator transitions, ACS Photonics 5, 4056 (2018).

[15] D. Lee, J. Lee, K. Song, F. Xue, S. Y. Choi, Y. Ma, J. Podkaminer, D. Liu, S. C. Liu, B. Chung, W. Fan, S. J. Cho, W. Zhou, J. Lee, L. Q. Chen, S. H. Oh, Z. Ma, and C. B. Eom, Sharpened $\mathrm{VO}_{2}$ phase transition via controlled release of epitaxial strain, Nano Lett. 17, 5614 (2017).

[16] J. del Valle, J. G. Ramírez, M. J. Rozenberg, and I. K. Schuller, Challenges in materials and devices for resistive-switching- based neuromorphic computing, J. Appl. Phys. 124, 211101 (2018).

[17] Y. Zhou and S. Ramanathan, Mott memory and neuromorphic devices, Proc. IEEE 103, 1289 (2015).

[18] P. Markov, R. E. Marvel, H. J. Conley, K. J. Miller, R. F. Haglund, and S. M. Weiss, Optically monitored electrical switching in $\mathrm{VO}_{2}$, ACS Photonics 2, 1175 (2015).

[19] P. Stoliar, J. Tranchant, B. Corraze, E. Janod, M.-P. P. Besland, F. Tesler, M. Rozenberg, and L. Cario, A leaky-integrate-andfire neuron analog realized with a Mott insulator, Adv. Funct. Mater. 27, 1604740 (2017).

[20] J. del Valle, N. M. Vargas, R. Rocco, P. Salev, Y. Kalcheim, M. Lee, C. Adda, P. Lapa, P. Y. Wang, L. Fratino, M. J. Rozenberg, and I. K. Schuller, Spatiotemporal characterization of the fieldinduced insulator-to-metal transition, Science 373, 907 (2021).

[21] S. Guénon, S. Scharinger, S. Wang, J. G. Ramírez, D. Koelle, R. Kleiner, and Ivan K. Schuller, Electrical breakdown in a $\mathrm{V}_{2} \mathrm{O}_{3}$ device at the insulator-to-metal transition, Europhys. Lett. 101, 57003 (2013).

[22] S. Kumar, M. D. Pickett, J. P. Strachan, G. Gibson, Y. Nishi, and R. S. Williams, Local temperature redistribution and structural transition during Joule-heating-driven conductance switching in $\mathrm{VO}_{2}$, Adv. Mater. 25, 6128 (2013).

[23] A. G. Shabalin, J. del Valle, N. Hua, M. J. Cherukara, M. V. Holt, I. K. Schuller, and O. G. Shpyrko, Nanoscale imaging and control of volatile and non-volatile resistive switching in $\mathrm{VO}_{2}$, Small 16, 2005439 (2020).

[24] M. Imada, A. Fujimori, and Y. Tokura, Metal-insulator transitions, Rev. Mod. Phys. 70, 1039 (1998).

[25] J. B. Torrance, P. Lacorre, A. I. Nazzal, E. J. Ansaldo, and C. Niedermayer, Systematic study of insulator-metal transitions in perovskites $R \mathrm{NiO}_{3}(R=\mathrm{Pr}, \mathrm{Nd}, \mathrm{Sm}, \mathrm{Eu})$ due to closing of charge-transfer gap, Phys. Rev. B 45, 8209 (1992).

[26] M. L. Medarde, Structural, magnetic and electronic properties of $R \mathrm{NiO}_{3}$ perovskites ( $R=$ rare earth), J. Phys. Condens. Matter. 9, 1679 (1997).

[27] S. Catalano, M. Gibert, J. Fowlie, J. Iñiguez, J. M. Triscone, and J. Kreisel, Rare-earth nickelates $\mathrm{RNiO}_{3}$ : Thin films and heterostructures, Rep. Prog. Phys. 81, 046501 (2018).

[28] J. Zaanen, G. A. Sawatzky, and J. W. Allen, Band Gaps and Electronic Structure of Transition-Metal Compounds, Phys. Rev. Lett. 55, 418 (1985).

[29] V. Bisogni, S. Catalano, R. J. Green, M. Gibert, R. Scherwitzl, Y. Huang, V. N. Strocov, P. Zubko, S. Balandeh, J. M. Triscone, G. Sawatzky, and T. Schmitt, Ground-state oxygen holes and the metal-insulator transition in the negative charge-transfer rare-earth nickelates, Nat. Commun. 7, 13017 (2016)

[30] I. I. Mazin, D. I. Khomskii, R. Lengsdorf, J. A. Alonso, W. G Marshall, R. M. Ibberson, A. Podlesnyak, M. J. Martínez-Lope, and M. M. Abd-Elmeguid, Charge Ordering as Alternative to Jahn-Teller Distortion, Phys. Rev. Lett. 98, 176406 (2007).

[31] A. Subedi, O. E. Peil, and A. Georges, Low-energy description of the metal-insulator transition in the rare-earth nickelates, Phys. Rev. B. 91, 075128 (2015).

[32] J. A. Alonso, J. L. García-Muñoz, M. T. Fernández-Díaz, M. A. G. Aranda, M. J. Martínez-Lope, and M. T. Casais, Charge Disproportionation in $R \mathrm{NiO}_{3}$ Perovskites: Simultaneous MetalInsulator and Structural Transition in $\mathrm{YNiO}_{3}$, Phys. Rev. Lett. 82, 3871 (1999). 
[33] M. Medarde, C. Dallera, M. Grioni, B. Delley, F. Vernay, J. Mesot, M. Sikora, J. A. Alonso, and M. J. MartínezLope, Charge disproportionation in $\mathrm{RNiO}_{3}$ perovskites $(R=$ rare earth) from high-resolution $\mathrm{x}$-ray absorption spectroscopy, Phys. Rev. B 80, 245105 (2009).

[34] M. Medarde, P. Lacorre, K. Conder, F. Fauth, and A. Furrer, Giant ${ }^{16} \mathrm{O}-{ }^{18} \mathrm{O}$ Isotope Effect on the Metal-Insulator Transition of $\mathrm{RNiO}_{3}$ Perovskites ( $R=$ Rare Earth), Phys. Rev. Lett. 80, 2397 (1998).

[35] A. D. Caviglia, R. Scherwitzl, P. Popovich, W. Hu, H. Bromberger, R. Singla, M. Mitrano, M. C. Hoffmann, S. Kaiser, P. Zubko, S. Gariglio, J.-M. Triscone, M. Först, and A. Cavalleri, Ultrafast Strain Engineering in Complex Oxide Heterostructures, Phys. Rev. Lett. 108, 136801 (2012).

[36] A. Mercy, J. Bieder, J. Íñiguez, and P. Ghosez, Structurally triggered metal-insulator transition in rare-earth nickelates, Nat. Commun. 8, 1677 (2017).

[37] N. Shukla, T. Joshi, S. Dasgupta, P. Borisov, D. Lederman, and S. Datta, Electrically induced insulator to metal transition in epitaxial $\mathrm{SmNiO}_{3}$ thin films, Appl. Phys. Lett. 105, 039901 (2014).

[38] H. Huang, Z. Luo, Y. Yang, Y. Yun, M. Yang, D. Meng, H. Wang, S. Hu, J. Bao, Y. Lu, and C. Gao, DC current induced metal-insulator transition in epitaxial $\mathrm{Sm}_{0.6} \mathrm{Nd}_{0.4} \mathrm{NiO}_{3} / \mathrm{LaAlO}_{3}$ thin film, AIP Adv. 4, 057102 (2014).

[39] S. Catalano, M. Gibert, V. Bisogni, O. E. Peil, F. He, R. Sutarto, M. Viret, P. Zubko, R. Scherwitzl, A. Georges, G. A. Sawatzky, T. Schmitt, and J. M. Triscone, Electronic transitions in strained $\mathrm{SmNiO}_{3}$ thin films, APL Mater. 2, 116110 (2014).

[40] D. Preziosi, L. Lopez-Mir, X. Li, T. Cornelissen, J. H. Lee, F. Trier, K. Bouzehouane, S. Valencia, A. Gloter, A. Barthélémy, and M. Bibes, Direct mapping of phase separation across the metal-insulator transition of $\mathrm{NdNiO}_{3}$, Nano Lett. 18, 2226 (2018).

[41] J. H. Lee, F. Trier, T. Cornelissen, D. Preziosi, K. Bouzehouane, S. S. Fusil, S. Valencia, and M. Bibes, Imaging and harnessing percolation at the metal-insulator transition of $\mathrm{NdNiO}_{3}$ nanogaps, Nano Lett. 19, 7801 (2019).

[42] M. Hepting, M. Minola, A. Frano, G. Cristiani, G. Logvenov, E. Schierle, M. Wu, M. Bluschke, E. Weschke, H.-U. Habermeier, E. Benckiser, M. Le Tacon, and B. Keimer, Tunable Charge and Spin Order in $\mathrm{PrNiO}_{3}$ Thin Films and Superlattices, Phys. Rev. Lett. 113, 227206 (2014).

[43] See Supplemental Material at http://link.aps.org/supplemental/ 10.1103/PhysRevB.104.165141 for details on the fabrication process, structural characterization, further transport measurements, and details of the simulations.

[44] C. Lichtensteiger, INTERACTIVEXRDFIT: A new tool to simulate and fit $\mathrm{x}$-ray diffractograms of oxide thin films and heterostructures, J. Appl. Crystallogr. 51, 1745 (2018).

[45] J. Shi, S. D. Ha, Y. Zhou, F. Schoofs, and S. Ramanathan, A correlated nickelate synaptic transistor, Nat. Commun. 4, 2676 (2013).

[46] L. Wang, Q. Zhang, L. Chang, L. You, X. He, K. Jin, L. Gu, H. Guo, C. Ge, Y. Feng, and J. Wang, Electrochemically driven giant resistive switching in perovskite nickelates heterostructures, Adv. Electron. Mater. 3, 1700321 (2017).

[47] J. C. Gonzalez-Rosillo, S. Catalano, I. Maggio-Aprile, M. Gibert, X. Obradors, A. Palau, and T. Puig, Nanoscale correla- tions between metal-insulator transition and resistive switching effect in metallic perovskite oxides, Small 16, 2001307 (2020).

[48] H. T. Zhang, T. J. Park, I. A. Zaluzhnyy, Q. Wang, S. N. Wadekar, S. Manna, R. Andrawis, P. O. Sprau, Y. Sun, Z. Zhang, C. Huang, H. Zhou, Z. Zhang, B. Narayanan, G. Srinivasan, N. Hua, E. Nazaretski, X. Huang, H. Yan, M. Ge et al., Perovskite neural trees, Nat. Commun. 11, 2245 (2020).

[49] A. Palau, A. Fernandez-Rodriguez, J. C. Gonzalez-Rosillo, X. Granados, M. Coll, B. Bozzo, R. Ortega-Hernandez, J. Suñé, N. Mestres, X. Obradors, and T. Puig, Electrochemical tuning of metal insulator transition and nonvolatile resistive switching in superconducting films, ACS Appl. Mater. Interfaces 10, 30522 (2018).

[50] S. Menzel, U. Böttger, M. Wimmer, and M. Salinga, Physics of the switching kinetics in resistive memories, Adv. Funct. Mater. 25, 6306 (2015).

[51] D. Ielmini, F. Nardi, C. Cagli, and A. L. Lacaita, Size-dependent retention time in NiO-based resistive-switching memories, IEEE Electron Device Lett. 31, 353 (2010).

[52] F. Tesler, C. Adda, J. Tranchant, B. Corraze, E. Janod, L. Cario, P. Stoliar, and M. Rozenberg, Relaxation of a Spiking Mott Artificial Neuron, Phys. Rev. Appl. 10, 054001 (2018).

[53] P. Stoliar, M. Rozenberg, E. Janod, B. Corraze, J. Tranchant, and L. Cario, Nonthermal and purely electronic resistive switching in a Mott memory, Phys. Rev. B 90, 045146 (2014).

[54] S. Catalano, M. Gibert, V. Bisogni, F. He, R. Sutarto, M. Viret, P. Zubko, R. Scherwitzl, G. A. Sawatzky, T. Schmitt, and J.-M. Triscone, Tailoring the electronic transitions of $\mathrm{NdNiO}_{3}$ films through $(111)_{\mathrm{pc}}$ oriented interfaces, APL Materials 3, 062506 (2015).

[55] J. Y. Zhang, H. Kim, E. Mikheev, A. J. Hauser, and S. Stemmer, Key role of lattice symmetry in the metal-insulator transition of $\mathrm{NdNiO}_{3}$ films, Sci. Rep. 6, 23652 (2016).

[56] A. Zimmers, L. Aigouy, M. Mortier, A. Sharoni, S. Wang, K. G. West, J. G. Ramirez, and I. K. Schuller, Role of Thermal Heating on the Voltage Induced Insulator-Metal Transition in $\mathrm{VO}_{2}$, Phys. Rev. Lett. 110, 056601 (2013).

[57] F. J. Morin, Oxides Which Show a Metal-to-Insulator Transition at the Neel Temperature, Phys. Rev. Lett. 3, 34 (1959).

[58] I. Valmianski, P. Y. Wang, S. Wang, J. G. Ramirez, S. Guénon, and I. K. Schuller, Origin of the current-driven breakdown in vanadium oxides: thermal versus electronic, Phys. Rev. B 98, 195144 (2018)

[59] G. Mazza, A. Amaricci, M. Capone, and M. Fabrizio, FieldDriven Mott Gap Collapse and Resistive Switch in Correlated Insulators, Phys. Rev. Lett. 117, 176401 (2016).

[60] P. Diener, E. Janod, B. Corraze, M. Querré, C. Adda, M. Guilloux-Viry, S. Cordier, A. Camjayi, M. Rozenberg, M. P. Besland, and L. Cario, How a dc Electric Field Drives Mott Insulators Out of Equilibrium, Phys. Rev. Lett. 121, 016601 (2018).

[61] Y. Kalcheim, A. Camjayi, J. del Valle, P. Salev, M. Rozenberg, and I. K. Schuller, Non-thermal resistive switching in Mott insulator nanowires, Nat. Commun. 11, 2985 (2020).

[62] A. Ronchi, P. Franceschini, P. Homm, M. Gandolfi, G. Ferrini, S. Pagliara, F. Banfi, M. Menghini, J.-P. Locquet, and C. Giannetti, Light-Assisted Resistance Collapse in a $\mathrm{V}_{2} \mathrm{O}_{3}$-Based Mott-Insulator Device, Phys. Rev. Appl. 15, 044023 (2021). 